\title{
Interactive Physics: A virtual library of simulations for use in Physics Undergraduate Teaching
}

\author{
Summary \\ This report outlines the design and deployment of a virtual (online) library of \\ interactive simulations (principally Java applets) designed to be used in \\ Undergraduate Physics teaching. The project was funded by an LTSN Physical \\ Sciences Project Development Grant in 2001.
}

Subject area: Physics

\begin{abstract}
Description
The use of Computers and Information Technology ( C\&IT') in teaching has genuine potential to enrich the teaching-learning experience, a fact that is borne out by its widespread, but not always effective, implementation across the HE sector. Our view of the role of C\&IT in the teaching of the Physical Sciences is that it should form part of a coherent learning environment, where technology is used to augment and enrich, rather than supplant, the more traditional methods of teaching. Our views are mirrored by the student community, who quite rightly expect a varied educational environment, firmly rooted in the face-to-face staff-student contact of a real (as opposed to 'virtual') University campus.
\end{abstract}

Our experience with the use of C\&IT thus far has demonstrated the truth in the old adage that a picture 'speaks a thousand words'. Better than a picture is a moving picture, and the incorporation of moving images into - via video or simulation - course material, textbooks, web resources etc is now becoming the norm. Our use of video clips and simulations during lectures has been generally well-received by our students and many find it a valuable aid to their understanding of concepts. The 'best of all worlds' situation is one in which the student has such a visual representation available, but in addition also has the ability to affect or 'steer' the simulation via their own intervention. In this way, the static presentation of a visual resource becomes an interactive experience.

Pedagogically, simulations can be immensely valuable if they are used in a manner based on a good understanding of student thought processes. They can enable students to form connections between the many and varied representations that we use in Physics, such as equations, diagrams, tables, vector plots etc. They can be used to cement the fact that the equations we use represent relationships between observations and measurements, that they are not just for the calculation of a quantity. Simulations can enable students to build mental pictures ('models') of physical systems and they can use these models as a vehicle to describe and convey their understanding to each other. Finally, simulations offer an engaging, hands-on, active learning experience that puts the student in control of events and can lead to a deeper learning.

With that rationale in mind, we have designed a virtual library to house interactive simulations (chiefly at present Java applets, but also Flash animations) that may be utilised in undergraduate Physics teaching. The key design feature is that of a virtual 
library, i.e. the applets themselves exist remotely (wherever they may be on the WWW) and we maintain placeholders to them and other resources (see below on use scenarios). The library is searchable in a variety of ways allowing different library users to locate resources in different ways. The following image is a screenshot of the applet library homepage

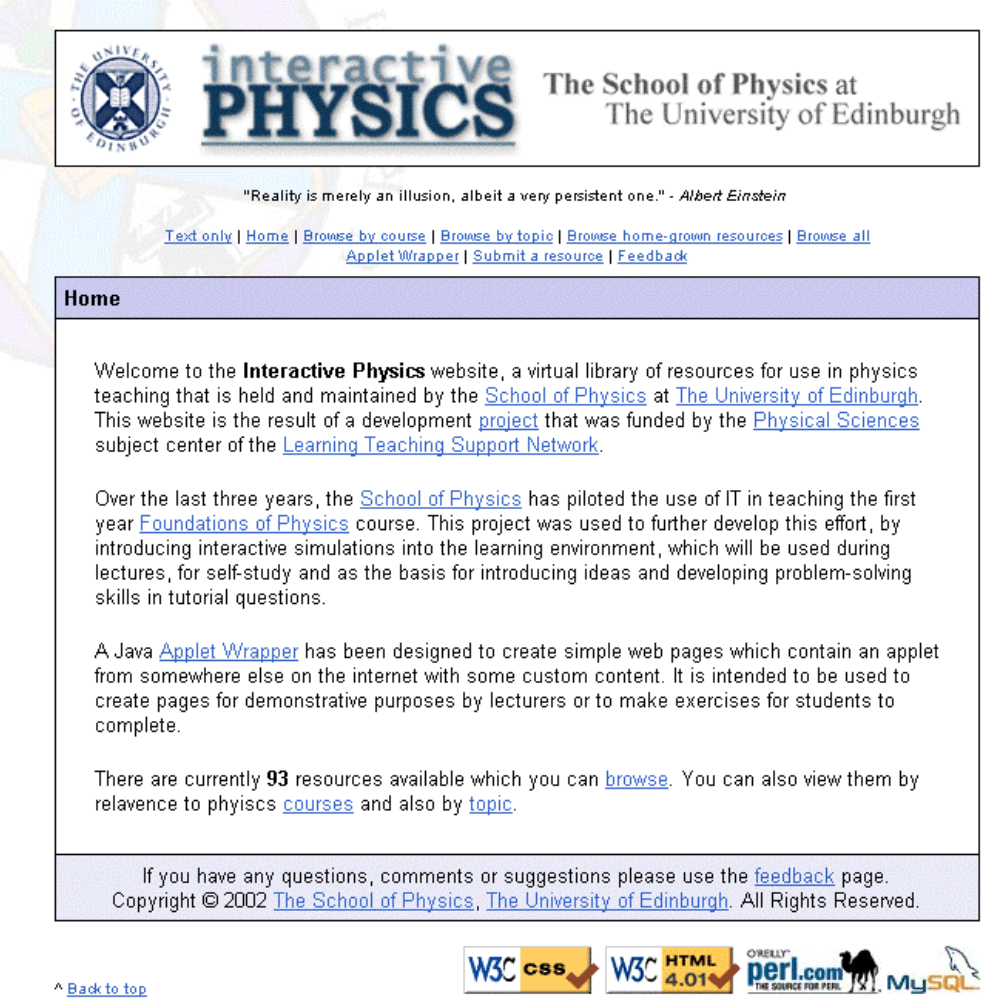

\section{Type of activity}

- $\quad$ The main technical details of the Interactive Physics Applet database are summarised below:

- $\quad$ The applet library (database) exists as a relational (MySQL) database

- $\quad$ Perl / cgi scripts (1200 lines) query the database on the fly, creating customised web pages, depending on how the user wants the information presented, e.g. ordered by course, by topic, full listing etc.

- $\quad$ The database is managed remotely using the ODBC protocol through Microsoft Access 2000. This allows modification of database content and design in a transparent way.

- $\quad$ The scripts and database design is fully documented to enable transfer of management responsibilities to different people. The separation of content (database) and presentation (script-generated Web pages) enables easy reuse of the project's components.

- $\quad$ The script automatically checks whether or not links are dead or alive and adjusts the displayed web page that is delivered to the user accordingly.

- $\quad$ Users are encouraged to report bad resources and can easily do so (e.g. bad links, inaccurate description etc).

- $\quad$ Newly-added resources are flagged so that users can see recent additions. Users can also suggest additions to the site. 
- $\quad$ An Applet Wrapper has been created, which the user views as a series of stepby-step instructions. This enables a non-expert to create high impact web pages with Applets embedded in them for offline use, without detailed knowledge of the html language. The following screenshot shows a wrapped applet without any detailed notes for use :

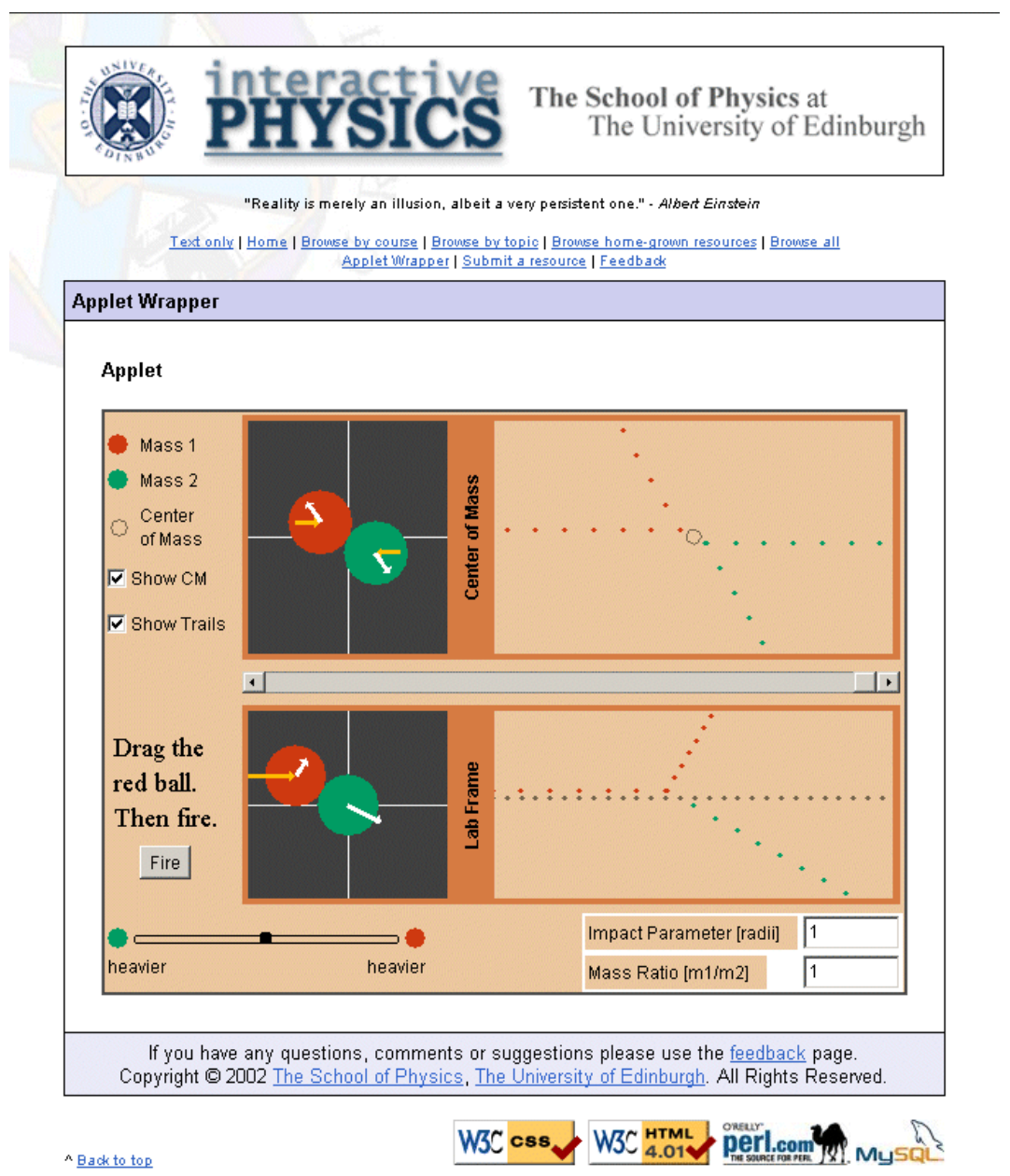

\section{Content covered}

Initially, the library's remit was to house resources for a foundation level course (1st year) in classical Physics (mechanics, dynamics, energy, kinematics and waves) taught at the University of Edinburgh. Although the library entries are geared towards more introductory material, there is a gradual percolation through to more advanced material, such as quantum theory, relativity etc.

The framework in place is such that there is provision to house applets / resources relevant to any aspect of undergraduate physics. The tools developed are inherently generic, and as such may be relatively easily transferred to other Physical sciences, e.g. Chemistry, Engineering etc. 


\title{
Application
}

There are principally two different user scenarios:

\section{The student}

The database is designed in such a way that students may search the database either by topic or by course, and view the relevant resources. Hyperlinks to the applet's source page enable the students to make use of other resources that may have been developed with the applet in question (e.g. exercises)

\section{The lecturer}

This approach to browsing the material is also available to lecturers. In addition, there is the capability to create a "wrapped-up" web page with the applet embedded in it, surrounded by learning material such as commentary, exercises and points to note, without a detailed knowledge of how to write raw html. This "applet wrapper" is available on the database and may be used to wrap up any applet in a series of easy to follow steps. The resulting web page is then able to be saved locally for use in a lecture, tutorial or for posting on a course website. For those with some knowledge of html, it may be possible (depending on how the applet was written) to customise the local ("wrapped up") version of the applet by altering parameters in the $<$ APPLET $>$ html tag.

\section{Further comments}

The future: This project has been extremely successful, with all of the aims being met on a very modest budget and a resource of real capability and usability for the academic community has been produced. Future development will include extending the number and range of applets in the database and more home-grown material to fill in what we perceive to be the gaps in material available remotely on the web. For this, we intend to seek further funding. The possibility of transferring this generically to other subjects (e.g. chemistry and engineering) is also being explored.

\author{
Author(s) \\ Simon Bates and Sean Wilson, \\ School of Physics, University of Edinburgh

\section{Contact details} \\ Dr Simon Bates, Lecturer in Computational Materials Physics \\ School of Physics \\ University of Edinburgh, \\ Edinburgh \\ EH9 3JZ \\ s.p.bates@ed.ac.uk
}

URL

http://www.ph.ed.ac.uk/applets 